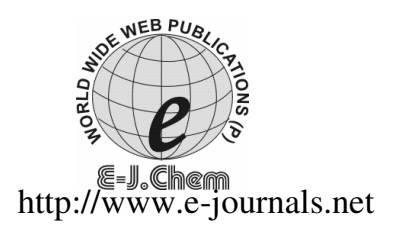

ISSN: 0973-4945; CODEN ECJHAO

E-Journal of Chemistry

2010, 7(4), 1498-1506

\title{
New Piperazine Derivatives Synthesized from Thio-Substituted Polyhalogeno-2-nitro-1,3-butadienes
}

\author{
S. GOKSIN AYDINLI* and CEMIL IBIS
}

\begin{abstract}
Faculty of Engineering, Department of Chemistry, Istanbul University, 34320 Avcilar, Istanbul, Turkey.

goksayd@istanbul.edu.tr
\end{abstract}

Received 25 December 2009; Accepted 20 February 2010

\begin{abstract}
It is known that polyhalogeno-nitro-1,3-butadienes are important starting materials for the synthesis of polyfunctionalized bioactive heterocycles. Novel $N, S$-substituted nitrobutadienes $(\mathbf{4 a - j})$ were synthesized from the reaction of the monothio-substituted nitrodiene derivatives (2a) and (2b) with some piperazine derivatives. These new compounds are stable and the structures of these products were characterized by spectroscopic data. The structure of the novel $N, S$-substituted nitrodiene compound $(\mathbf{4 g})$ synthesized in this study was also elucidated by single crystal $\mathrm{x}$-ray analysis.
\end{abstract}

Keywords: Monothio-substituted nitrodiene, Piperazine, $N, S$-substituted nitrobutadiene, $\mathrm{X}$-ray analysis.

\section{Introduction}

It is worthwhile to note that polyhalogeno-1,3-butadienes bearing at least one nitro group are in general valuable starting materials for the synthesis of polyfunctionalized bioactive heterocycles. In recent investigations, the synthesis of some polyhalogenonitroalkenes and nitrodienes having heterocyclic ring has been reported. It has been described formerly some preparation procedures for $\mathrm{N}, \mathrm{N}-,, \mathrm{N}, \mathrm{S}-, \mathrm{N}, \mathrm{O}-$ and $\mathrm{O}, \mathrm{S}$ substituted polyhalogeno-1,3-butadienes ${ }^{1-7}$. It has been reported before in some patents $^{8,9}$ and in other publications that mixed halogenobutadienes possess a broad of useful properties ${ }^{4}$ and it is known that some heterocyclic amine derivatives like piperazine salts also have been incorporated into a wide variety of clinical chemistry as gene transfer reactions ${ }^{10-12}$, spasmolytic ${ }^{13}$, anthelmintic ${ }^{14}$ or germicial $^{15}$ activities. $^{-}$

We have previously synthesized $N, S$-substituted nitrobutadienes from the reaction of some thio-substituted nitrobutadienes with heterocyclic amine derivatives ${ }^{16-25}$. In the present paper, 1-(1,3,4,4-tetrachloro-2-nitro-buta-1,3-dienylsulfanyl)-octane $(\mathbf{2 a})^{26}$ and $(1,3,4,4-$ tetrachloro-2-nitro-buta-1,3-dienylsulfanyl)-cyclohexane $(\mathbf{2 b})^{27}$ serves as starting material for the new $N, S$-substituted nitrodienes. $\mathbf{2 a}$ and $\mathbf{2 b}$ can be synthesized from the reaction of 
pentachloro-2-nitro-1,3-butadiene (1) $\left(\mathrm{Cl}_{2} \mathrm{C}=\mathrm{CCI}-\mathrm{C}\left(\mathrm{NO}_{2}\right)=\mathrm{CCI}_{2}\right)$ and suitable thiols. As a part of our studies on polyhalogeno nitrobutadienes, we also synthesized and characterized new halogenated diene-piperazine compounds that these novel $N, S$-substituted nitrodienes $(\mathbf{4 a}-\mathbf{j})$ were obtained from the reaction of monothio-substituted nitrodienes (2a) and (2b) with some piperazine derivatives (Figure 1).

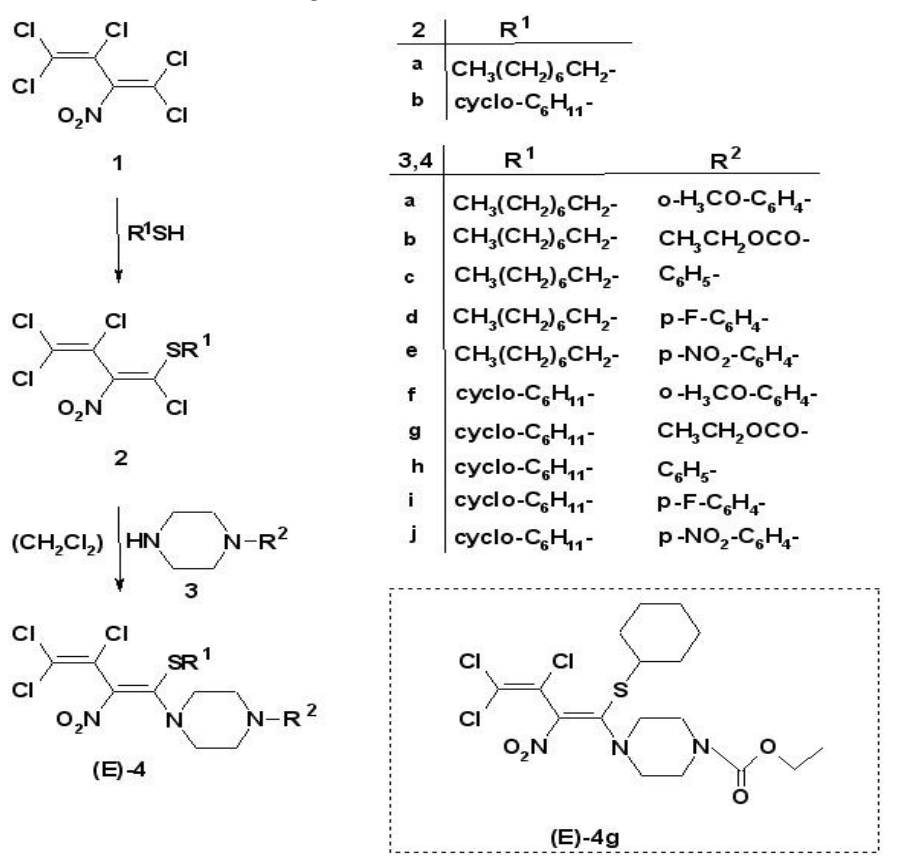

Figure 1. Synthesis of substituted 2-nitrodienes

The structure of 4-(3,4,4-trichloro-1-cyclohexylsulfanyl-2-nitro-buta-1,3-dienyl)piperazine-1-carboxylic acid ethyl ester $(\mathbf{4 g})$.

\section{Experimental}

Melting points were determined with a Buchi B-540 apparatus and were uncorrected. Elemental analyses were carried out on a Thermo Finnigan Flash EA 1112 analyzer. Infrared spectral data were obtained on a Shimadzu FTIR-8101 spectrometer in KBr discs. ${ }^{1} \mathrm{H}$ and ${ }^{13} \mathrm{C}$ NMR spectra were obtained on a VarianUNITY INOVA spectrometer. Mass spectra were obtained on a Finnigan LCQ Advantage Max. LC/MS. TLC was done on Merck TLC plates (aluminium backed) silica gel 60 (Merck). Column chromatography was carried out on Silica gel 60 (Merck). All chemicals were reagent grade and used without further purification and moisture was excluded from the glass apparatus using $\mathrm{CaCl}_{2}$ drying tubes.

\section{Synthesis}

\section{Preparation of compounds $(\mathbf{4} \boldsymbol{a}-\boldsymbol{j})$}

As starting materials, 1-(1,3,4,4-tetrachloro-2-nitro-buta-1,3-dienylsulfanyl)-octane (2a) and (1,3,4,4-tetrachloro-2-nitro-buta-1,3-dienylsulfanyl)-cyclohexane (2b) were prepared from Pentachloro-2-nitro-1,3-butadiene $(\mathbf{1})^{26-28}$. To a solution of $0.4 \mathrm{~g}(1.05 \mathrm{mmol}) \mathbf{2 a}$ or $0.4 \mathrm{~g}(1.14$ $\mathrm{mmol}) \mathbf{2 b}$ in $10 \mathrm{~mL}$ of dichloromethane as solvent was added in equimolar amount of the 
respective piperazine derivatives in $10 \mathrm{~mL}$ of dichloromethane with vigorous stirring at room temperature until completion of the reaction. The mixture was stirred for $4 \mathrm{~h}$ and the crude products were extracted with $\mathrm{CHCl}_{3}(3 \times 50 \mathrm{~mL})$. The combined organic phases were washed with $\mathrm{H}_{2} \mathrm{O}(2 \times 30 \mathrm{~mL})$ and dried over anhydrous $\mathrm{CaCl}_{2}$ or $\mathrm{MgSO}_{4}$ in vacuo. The products were either crystallized or purified by column chromatography over silica gel.

\section{1-(2-Methoxy-phenyl)-4-(3,4,4-trichloro-2-nitro-1-octylsulfanyl-buta-1,3-dienyl)- piperazine (4a)}

Yield: $0.300 \mathrm{~g}(53 \%)$; Oil, $\mathrm{R}_{\mathrm{f}}\left(\mathrm{CH}_{2} \mathrm{Cl}_{2}\right)$ : 0.22; IR (film) $=2855,2926 \mathrm{~cm}^{-1} \quad(\mathrm{C}-\mathrm{H}), 1594$ $(\mathrm{C}=\mathrm{C}), 1277,1504\left(\mathrm{C}-\mathrm{NO}_{2}\right) ;{ }^{1} \mathrm{H}$ NMR $\left(500 \mathrm{MHz}, \mathrm{CDCl}_{3}, \delta / \mathrm{ppm}\right): 0.76-1.13(\mathrm{~m}, 3 \mathrm{H}$, $\left.\mathrm{CH}_{3}\right), 1.18-1.20\left(\mathrm{~m}, 12 \mathrm{H},\left(\mathrm{CH}_{2}\right)_{6}\right), 2.87\left(\mathrm{t}, \mathrm{J}=7.32,2 \mathrm{H}, \mathrm{SCH}_{2}\right), 3.13\left(\mathrm{bs}, 3 \mathrm{H}, \mathrm{OCH}_{3}\right)$, 3.64-3.84 (m, 8H, piper-H), 6.80-7.19 (m, 4H, arom-H); ${ }^{13} \mathrm{C} \mathrm{NMR} \mathrm{(125} \mathrm{MHz,} \mathrm{CDCl}_{3}$, $\delta / \mathrm{ppm}): 09.9,13.0\left(\mathrm{CH}_{3}\right), 21.5,27.6,27.9,28.0,28.7,30.6,34.5\left(\mathrm{CH}_{2}\right), 52.6,54.5(\mathrm{~N}-$ $\mathrm{CH}_{2}$ ), 110.7, 117.0, 117.5, 120.1 (arom-CH), 123.1, 123.4 (arom-C), 126.0, 138.5, 151.3, 168.2 (butad-C); APCI-MS $m / z(\%): 537.97\left[\mathrm{M}^{+}\right]$(100), 537.01 (28.20), 538.99 (26.76); $\mathrm{C}_{23} \mathrm{H}_{32} \mathrm{Cl}_{3} \mathrm{~N}_{3} \mathrm{O}_{3} \mathrm{~S}$ (536.95).

4-(3,4,4-Trichloro-2-nitro-1-octylsulfanyl-buta-1,3-dienyl)-piperazine-1-carboxylic acid ethyl ester (4b)

Yield: $0.331 \mathrm{~g}(63 \%)$; Oil, $\mathrm{R}_{\mathrm{f}}\left(\mathrm{CH}_{2} \mathrm{Cl}_{2}\right)$ : 0.35; IR (film) = 2856, $2927 \mathrm{~cm}^{-1}(\mathrm{C}-\mathrm{H}), 1531$ $(\mathrm{C}=\mathrm{C}), 1704(\mathrm{C}=\mathrm{O}), 1274,1428\left(\mathrm{C}-\mathrm{NO}_{2}\right) ;{ }^{1} \mathrm{H}$ NMR (500 MHz, $\left.\mathrm{CDCl}_{3}, \delta / \mathrm{ppm}\right): 0.86-1.26$ $\left(\mathrm{m}, 3 \mathrm{H}, \mathrm{CH}_{3}\right), 1.28-1.70\left(\mathrm{~m}, 15 \mathrm{H},\left(\mathrm{CH}_{2}\right)_{6}, \mathrm{OCH}_{2} \mathrm{CH}_{3}\right), 2.96\left(\mathrm{t}, \mathrm{J}=7.32,2 \mathrm{H}, \mathrm{SCH}_{2}\right), 3.45-$ $3.83\left(\mathrm{~m}, 8 \mathrm{H}\right.$, piper-H), 4.19 (q, J = 7.32, 2H, OCH $\left.\mathrm{CH}_{3}\right) ;{ }^{13} \mathrm{C} \mathrm{NMR}\left(125 \mathrm{MHz}, \mathrm{CDCl}_{3}\right.$, $\delta$ / ppm): 13.0, $13.5\left(\mathrm{CH}_{3}\right), 21.5,27.6,27.9,28.1,28.7,30.6,34.5,42.2\left(\mathrm{CH}_{2}\right), 51.8,61.0$ $\left(\mathrm{N}-\mathrm{CH}_{2}\right), 117.8(\mathrm{C}=\mathrm{O}), 123.8,125.7,154.0,168.6$ (butad-C); APCI-MS $\mathrm{m} / \mathrm{z}(\%): 501.95$ $\left[\mathrm{M}^{+}\right]$(100), 503.11 (32.91), 503.94 (77.37); $\mathrm{C}_{19} \mathrm{H}_{30} \mathrm{Cl}_{3} \mathrm{~N}_{3} \mathrm{O}_{4} \mathrm{~S}$ (502.89). Calcd. C 45.38, $\mathrm{H}$ 6.01, N 8.36; Found C 45.30, H 5.84, N 8.23.

\section{1-Phenyl-4-(3,4,4-trichloro-2-nitro-1-octylsulfanyl-buta-1,3-dienyl)-piperazine (4c)}

Yield: $0.342 \mathrm{~g}(64 \%)$; Oil, $\mathrm{R}_{\mathrm{f}}\left(\mathrm{CH}_{2} \mathrm{Cl}_{2}\right)$ : 0.48; IR (film) $=2855,2926 \mathrm{~cm}^{-1}(\mathrm{C}-\mathrm{H}), 1600$ $(\mathrm{C}=\mathrm{C}), 1277,1531\left(\mathrm{C}-\mathrm{NO}_{2}\right) ;{ }^{1} \mathrm{H}$ NMR $\left(500 \mathrm{MHz}, \mathrm{CDCl}_{3}, \delta / \mathrm{ppm}\right): 0.84-0.92\left(\mathrm{~m}, 3 \mathrm{H}, \mathrm{CH}_{3}\right)$, 1.26-1.70 (m, 12H, $\left.\left(\mathrm{CH}_{2}\right)_{6}\right), 2.98\left(\mathrm{t}, \mathrm{J}=7.32,2 \mathrm{H}, \mathrm{SCH}_{2}\right), 3.26-3.89(\mathrm{~m}, 8 \mathrm{H}$, piper-H), 6.93$7.39(\mathrm{~m}, 5 \mathrm{H}$, arom-H$) ;{ }^{13} \mathrm{C} \mathrm{NMR}\left(125 \mathrm{MHz}, \mathrm{CDCl}_{3}, \delta / \mathrm{ppm}\right): 13.0\left(\mathrm{CH}_{3}\right), 21.5,27.6,27.9$, 28.0, 28.7, 30.6, $34.6\left(\mathrm{CH}_{2}\right), 48.4,52.0\left(\mathrm{~N}^{-\mathrm{CH}_{2}}\right), 109.6,115.7,116.4,117.3$ (arom-CH), 120.3, 123.6 (arom-C), 125.8, 128.4, 148.7, 168.3 (butad-C); APCI-MS m/z (\%): 507.97 $\left[\mathrm{M}^{+}\right]$(100), 507.02 (31.55), 505.94 (94.01); $\mathrm{C}_{22} \mathrm{H}_{30} \mathrm{Cl}_{3} \mathrm{~N}_{3} \mathrm{O}_{2} \mathrm{~S}$ (506.93). Calcd. C 52.13, $\mathrm{H}$ 5.97, N 8.29; Found C 52.34, H 6.12, N 8.33.

\section{1-(4-Fluoro-phenyl)-4-(3,4,4-trichloro-2-nitro-1-octylsulfanyl-buta-1,3-dienyl)- piperazine $\mathbf{( 4 d )}$}

Yield: $0.345 \mathrm{~g}$ (63\%); Oil, $\mathrm{R}_{\mathrm{f}}\left(\mathrm{CH}_{2} \mathrm{Cl}_{2}\right)$ : 0.43; IR (film) = 2855, $2926 \mathrm{~cm}^{-1}(\mathrm{C}-\mathrm{H}), 1596$ $(\mathrm{C}=\mathrm{C}), 1278,1520\left(\mathrm{C}-\mathrm{NO}_{2}\right) ;{ }^{1} \mathrm{H}$ NMR $\left(500 \mathrm{MHz}, \mathrm{CDCl}_{3}, \delta / \mathrm{ppm}\right): 0.86-0.92\left(\mathrm{~m}, 3 \mathrm{H}, \mathrm{CH}_{3}\right)$, 1.26-1.73 (m, 12H, $\left.\left(\mathrm{CH}_{2}\right)_{6}\right), 2.94-3.14\left(\mathrm{~m}, 2 \mathrm{H}, \mathrm{SCH}_{2}\right), 3.25-3.99(\mathrm{~m}, 8 \mathrm{H}$, piper-H), 6.90-7.26 (m, 4H, arom-H); ${ }^{13} \mathrm{C}$ NMR (125 MHz, $\left.\mathrm{CDCl}_{3}, \delta / \mathrm{ppm}\right): 14.0\left(\mathrm{CH}_{3}\right), 22.6,28.7,28.9,29.0$, 31.7, 35.6, $39.3\left(\mathrm{CH}_{2}\right), 50.5,53.0\left(\mathrm{~N}_{-} \mathrm{CH}_{2}\right), 115.8,116.0,118.6,118.8$ (arom-CH), 124.8, 126.7 (arom-C), 146.4, 157.1, 159.0, 169.2 (butad-C); APCI-MS m/z (\%): $525.91\left[\mathrm{M}^{+}\right]$ (100), 526.78 (21.97), 525.08 (22.48), 523.90 (83.59); $\mathrm{C}_{22} \mathrm{H}_{29} \mathrm{Cl}_{3} \mathrm{FN}_{3} \mathrm{O}_{2} \mathrm{~S}$ (524.92). Calcd. C 50.34, H 5.57, N 8.01; Found C 49.72, H 5.76, N 7.71. 
1-(4-Nitro-phenyl)-4-(3,4,4-trichloro-2-nitro-1-octylsulfanyl-buta-1,3-dienyl)piperazine (4e)

Yield: $0.301 \mathrm{~g}(52 \%)$; m.p.:156-157 ${ }^{0} \mathrm{C} . \mathrm{R}_{\mathrm{f}}\left(\mathrm{CH}_{2} \mathrm{Cl}_{2}\right)$ : 0.29; IR $(\mathrm{KBr})=2854,2925 \mathrm{~cm}^{-1}(\mathrm{C}$ $\mathrm{H}), 1600(\mathrm{C}=\mathrm{O}), 1310,1520\left(\mathrm{C}-\mathrm{NO}_{2}\right) ;{ }^{1} \mathrm{H}$ NMR $\left(500 \mathrm{MHz}, \mathrm{CDCl}_{3}, \delta / \mathrm{ppm}\right): 0.86-0.89(\mathrm{~m}$, $\left.3 \mathrm{H}, \mathrm{CH}_{3}\right), 1.26-1.74\left(\mathrm{~m}, 12 \mathrm{H},\left(\mathrm{CH}_{2}\right)_{6}\right), 2.98-3.13\left(\mathrm{~m}, 2 \mathrm{H}, \mathrm{SCH}_{2}\right), 3.14-3.84(\mathrm{~m}, 8 \mathrm{H}$, piper-H), 6.81-8.18 (m, 4H, arom-H); ${ }^{13} \mathrm{C}$ NMR (125 MHz, $\left.\mathrm{CDCl}_{3}, \delta / \mathrm{ppm}\right): 14.0\left(\mathrm{CH}_{3}\right), 22.6,28.7$, 28.9, 29.0, 29.7, 31.7, $35.7\left(\mathrm{CH}_{2}\right), 46.6,51.9\left(\mathrm{~N}_{-} \mathrm{CH}_{2}\right), 113.1,115.2$, 118.7, 118.9(arom$\mathrm{CH}$ ), 125.1, 126.0 (arom-C), 126.5, 139.8, 153.6, 169.5 (butad-C); APCI-MS m/z (\%): $552.95\left[\mathrm{M}^{+}\right]$(100), 552.00 (20.12), 550.97 (91.90); $\mathrm{C}_{22} \mathrm{H}_{29} \mathrm{Cl}_{3} \mathrm{~N}_{4} \mathrm{O}_{4} \mathrm{~S}$ (551.92). Calcd. C 47.88, H 5.30, N 10.15; Found C 47.63, H 5.82, N 9.60.

1-(2-Methoxy-phenyl)-4-(3,4,4-trichloro-1-cyclohexylsulfanyl-2-nitro-buta-1,3dienyl)-piperazine (4f)

Yield: $0.390 \mathrm{~g}(68 \%)$; m.p.: $135-136{ }^{0} \mathrm{C} . \mathrm{R}_{\mathrm{f}}\left(\mathrm{CH}_{2} \mathrm{Cl}_{2}\right): 0.37$; IR $(\mathrm{KBr})=2849,2923 \mathrm{~cm}^{-1}(\mathrm{C}-\mathrm{H})$, $1585(\mathrm{C}=\mathrm{C}), 1275,1537\left(\mathrm{C}-\mathrm{NO}_{2}\right) ;{ }^{1} \mathrm{H}$ NMR $\left(500 \mathrm{MHz}, \mathrm{CDCl}_{3}, \delta / \mathrm{ppm}\right): 1.17-1.98(\mathrm{~m}$, $11 \mathrm{H}$, cyclohex-H), $3.16\left(\mathrm{bs}, 3 \mathrm{H}, \mathrm{OCH}_{3}\right), 3.36-4.40(\mathrm{~m}, 8 \mathrm{H}$, piper-H), 6.84-7.24 (m, 4H, arom-H); ${ }^{13} \mathrm{C}$ NMR (125 MHz, $\left.\mathrm{CDCl}_{3}, \delta / \mathrm{ppm}\right): 13.4(\mathrm{CH} 3), 24.1,24.9,48.0,49.4\left(\mathrm{CH}_{2}\right)$, 52.7, $54.5\left(\mathrm{~N}-\mathrm{CH}_{2}\right), 110.6,117.4,120.1,122.9$ (arom-CH), 123.6, 125.9 (arom-C), 129.8, 138.6, 151.2, 166.6 (butad-C); APCI-MS m/z (\%) : $507.93\left[\mathrm{M}^{+}\right]$(100), 506.95 (24.29), 505.94 (89.41); $\mathrm{C}_{21} \mathrm{H}_{26} \mathrm{Cl}_{3} \mathrm{~N}_{3} \mathrm{O}_{3} \mathrm{~S}$ (506.88).

\section{4-(3,4,4-Trichloro-1-cyclohexylsulfanyl-2-nitro-buta-1,3-dienyl)-piperazine-1-carboxylic} acid ethyl ester $\mathbf{( 4 g})$

Yield: $0.282 \mathrm{~g}(52 \%)$; m.p.:132-133 ${ }^{0} \mathrm{C} . \mathrm{R}_{\mathrm{f}}\left(\mathrm{CH}_{2} \mathrm{Cl}_{2}\right)$ : 0.10; IR $(\mathrm{KBr})=2858,2941 \mathrm{~cm}^{-1}$ $(\mathrm{C}-\mathrm{H}), 1698(\mathrm{C}=\mathrm{O}), 1600(\mathrm{C}=\mathrm{C}), 1286,1524\left(\mathrm{C}-\mathrm{NO}_{2}\right) ;{ }^{1} \mathrm{H}$ NMR $\left(500 \mathrm{MHz}, \mathrm{CDCl}_{3}, \delta /\right.$ ppm): 1.24-1.25 (m, 3H, $\left.\mathrm{CH}_{3}\right), 1.26-2.00(\mathrm{~m}, 11 \mathrm{H}$, cyclohex-H), 3.37-4.16 (m, 8H, piper-H), $4.17\left(\mathrm{q}, \mathrm{J}=7.32,2 \mathrm{H}, \mathrm{OCH}_{2}\right) ;{ }^{13} \mathrm{C} \mathrm{NMR}\left(125 \mathrm{MHz}, \mathrm{CDCl}_{3}, \delta / \mathrm{ppm}\right): 14.6$ $\left(\mathrm{CH}_{3}\right), 25.9,29.7,33.7,43.3,49.2\left(\mathrm{CH}_{2}\right), 52.9,62.1\left(\mathrm{~N}_{-} \mathrm{CH}_{2}\right), 119.3(\mathrm{C}=\mathrm{O}), 125.1$, 126.6, 155.1, 168.1 (butad-C); APCI-MS m/z (\%) : $473.89\left[\mathrm{M}^{+}\right]$(100), 472.94 (23.68), 471.88 (96.90); $\mathrm{C}_{17} \mathrm{H}_{24} \mathrm{Cl}_{3} \mathrm{~N}_{3} \mathrm{O}_{4} \mathrm{~S}$ (472.82). Calcd. C 43.19, H 5.12, N 8.89; Found $\mathrm{C}$ 42.74, H 5.65, N 9.39.

\section{1-Phenyl-4-(3,4,4-trichloro-1-cyclohexylsulfanyl-2-nitro-buta-1,3-dienyl)-piperazine (4h)}

Yield: $0.275 \mathrm{~g}(51 \%)$; m.p.: $138-139{ }^{0} \mathrm{C} . \mathrm{R}_{\mathrm{f}}\left(\mathrm{CH}_{2} \mathrm{Cl}_{2}\right)$ : 0.32; IR $(\mathrm{KBr})=2825,2856$, $2942 \mathrm{~cm}^{-1}(\mathrm{C}-\mathrm{H}), 1599(\mathrm{C}=\mathrm{C}), 1259,1532\left(\mathrm{C}-\mathrm{NO}_{2}\right) ;{ }^{1} \mathrm{H}$ NMR $\left(500 \mathrm{MHz}, \mathrm{CDCl}_{3}, \delta /\right.$ ppm): 1.10-2.01 (m, 11H, cyclohex-H), 3.20-4.20 (m, 8H, piper-H), 6.91-7.31 (m, 5H, arom-H); ${ }^{13} \mathrm{C}$ NMR (125 MHz, $\left.\mathrm{CDCl}_{3}, \delta / \mathrm{ppm}\right): 24.1,24.9,30.5,48.2\left(\mathrm{CH}_{2}\right), 48.4,52.1$ $\left(\mathrm{N}-\mathrm{CH}_{2}\right), 115.6,116.2,117.6,118.3(\operatorname{arom}-\mathrm{CH}), 120.1,123.8$ (arom-C), 125.7, 128.4, 148.9, 166.8 (butad-C); APCI-MS $m / z(\%): 476.05\left[\mathrm{M}^{+}\right](100), 477.10$ (18.83), 477.97 (90.51); $\mathrm{C}_{20} \mathrm{H}_{24} \mathrm{Cl}_{3} \mathrm{~N}_{3} \mathrm{O}_{2} \mathrm{~S}$ (476.86). Calcd. C 50.38, H 5.07, N 8.81; Found C 49.93, $\mathrm{H}$ $4.89, \mathrm{~N} 8.68$.

1-(4-Fluoro-phenyl)-4-(3,4,4-trichloro-1-cyclohexylsulfanyl-2-nitro-buta-1,3dienyl)-piperazine (4i)

Yield: $0.287 \mathrm{~g}$ (51\%); Oil, $\mathrm{R}_{\mathrm{f}}\left(\mathrm{CH}_{2} \mathrm{Cl}_{2}\right)$ : 0.35; IR (film) $=2853,2925 \mathrm{~cm}^{-1}(\mathrm{C}-\mathrm{H}), 1508$ $(\mathrm{C}=\mathrm{C}), 1282,1456\left(\mathrm{C}-\mathrm{NO}_{2}\right) ;{ }^{1} \mathrm{H}$ NMR $\left(500 \mathrm{MHz}, \mathrm{CDCl}_{3}, \delta / \mathrm{ppm}\right): 0.84-2.02(\mathrm{~m}, 11 \mathrm{H}$, cyclohex-H), 2.04-4.40 (m, 8H, piper-H), 6.87-7.01 (m, 4H, arom-H); ${ }^{13} \mathrm{C}$ NMR (125 MHz, 
$\left.\mathrm{CDCl}_{3}, \delta / \mathrm{ppm}\right): 24.1,28.6,31.8,48.2\left(\mathrm{CH}_{2}\right), 49.5,52.1\left(\mathrm{~N}-\mathrm{CH}_{2}\right), 114.8,114.9,117.6$, 117.7 (arom-CH), 123.9, 125.7 (arom-C), 145.6, 156.0, 157.9, 166.7 (butad-C); APCIMS m/z (\%): $495.94\left[\mathrm{M}^{+}\right]$(100), 494.92 (23.74), 493.90 (89.21); $\mathrm{C}_{20} \mathrm{H}_{23} \mathrm{Cl}_{3} \mathrm{FN}_{3} \mathrm{O}_{2} \mathrm{~S}$ (494.85).

1-(4-Nitro-phenyl)-4-(3,4,4-trichloro-1-cyclohexylsulfanyl-2-nitro-buta-1,3-dienyl)piperazine $(\mathbf{4 j})$

Yield: $0.322 \mathrm{~g}(54 \%)$; m.p.: $187-188{ }^{\circ} \mathrm{C} . \mathrm{R}_{\mathrm{f}}\left(\mathrm{CH}_{2} \mathrm{Cl}_{2}\right)$ : 0.25; IR $(\mathrm{KBr})=2852,2928 \mathrm{~cm}^{-1}(\mathrm{C}$ $\mathrm{H}), 1596(\mathrm{C}=\mathrm{C}), 1320,1523\left(\mathrm{C}_{-} \mathrm{NO}_{2}\right) ;{ }^{1} \mathrm{H} \mathrm{NMR}\left(500 \mathrm{MHz}, \mathrm{CDCl}_{3}, \delta / \mathrm{ppm}\right): 1.20-2.02(\mathrm{~m}$, $11 \mathrm{H}$, cyclohex-H), 3.44-3.96 (m, 8H, piper-H), $6.83(\mathrm{~d}, \mathrm{~J}=9.28,2 \mathrm{H}$, arom-H), $8.13(\mathrm{~d}, \mathrm{~J}=$ 9.28, 2H, arom-H); $\left.{ }^{13} \mathrm{C} \mathrm{NMR} \mathrm{(125} \mathrm{MHz,} \mathrm{CDCl}_{3}, \delta / \mathrm{ppm}\right): 24.1,24.9,45.1,45.6\left(\mathrm{CH}_{2}\right)$,

48.4, $51.1\left(\mathrm{~N}_{-} \mathrm{CH}_{2}\right), 112.1,112.6,118.0,124.2$ (arom-CH), 124.9, 125.0 (arom-C), 125.5, 138.6, 152.6, 167.1 (butad-C); APCI-MS m/z (\%): $522.92\left[\mathrm{M}^{+}\right]$(100), 523.84 (28.85), 521.97 (14.12), 521.08 (71.38); $\mathrm{C}_{20} \mathrm{H}_{23} \mathrm{Cl}_{3} \mathrm{~N}_{4} \mathrm{O}_{4} \mathrm{~S}$ (521.85). Calcd. C 46.03, H 4.44, N 10.74; Found C 45.82, H 4.58, N 11.12.

Activated diene systems are widely used in organic synthesis for preparing diverse functionality substituted organic compounds, as well as convenient starting materials for syntheses on the basis of nucleophilic substitution reactions. Due to their stepped reactivity in substitution reactions, nitro-substituted polyhalogeno-1,3-butadienes have proven to be valuable synthetic precursors for a variety of polyfunctionalized bioactive heterocycles. The activated terminal carbon atom $\mathrm{C}-1$ of the nitrodichlorovinyl moiety in nitrodienes allows for an attack by different nucleophiles in $S_{N} V$ in processes. The direction of the $S_{N} V$ in process would depend on a number of factors: on nucleophile reactivity, on the character of the substituents in the nitrodiene chain on reactions conditions, etc $^{29-33}$.

The structures of the products were established on the basis of their IR, NMR and Mass spectra. In the IR spectra, the nitro group gives rise to absorption bands in the regions 1250-1320 (symmetric vibrations) and 1420-1540 $\mathrm{cm}^{-1}$ (antisymmetric vibrations). Vibrations of the $\mathrm{C}=\mathrm{C}$ bonds are characterized by absorption bands at $1530-1615 \mathrm{~cm}^{-1}$. Stretching vibrations of the carbonyl groups in compounds $\mathbf{4 b}$ and $\mathbf{4 g}$ were characterized by strong absorption bands at 1704 and $1698 \mathrm{~cm}^{-1}$, respectively.

In the ${ }^{1} \mathrm{H}$ NMR spectra of compounds $\mathbf{4 a - j}$, piperazine hydrogens appear as multiplet in the region $2.00-4.50 \mathrm{ppm}$ and in compounds $\mathbf{4} \mathbf{f}-\mathbf{j}$, cyclohexane hydrogens appear in the region $0.80-2.10 \mathrm{ppm}$ as multiplet.

\section{Crystallographic measurements}

The stereochemistry of the nitrodiene compound $\mathbf{4 g}$ was also confirmed by the result of a single crystal x-ray structure determination. Experimental details for data collection and structure refinement are summarized in Table 1. ORTEP diagram of the molecular structure of $\mathbf{4 g}$ in the crystal with atom numbering scheme is shown in Figure 2 and selected bond lengths and angles can be found in Table 2. The molecule packing diagram for compound $\mathbf{4 g}$ is shown in Figure 3 as a projection along a-axis. X-Ray structural analysishave shown that the nitrodiene $\mathbf{4 g}$ is $E$ isomer with a non-planar and close to cisoid configuration. Some $E$ - and $Z$-isomer thiosubstitue nitrodiene compounds have been reported ${ }^{34-41}$ that the observed values in compound $\mathbf{4 g}$ is consistent with the corresponding values in these similar compounds. 
The nitro groups show virtually coplanarity with the double bond (torsion angle $\left.\mathrm{O} 2 \mathrm{~N} 1 \mathrm{C} 3 \mathrm{C} 4-14.6(2)^{0}\right)$ and also the single bond (torsion angle O1N1C3C2 $\left.-10.7(2)^{0}\right)$. The $\mathrm{C}-\mathrm{C}$ bond lengths of the butadiene chain are 1.326(2), 1.454(2) and 1.400(2) $\AA$ for $\mathrm{C} 2-\mathrm{C} 1$, $\mathrm{C} 3-\mathrm{C} 2$ and $\mathrm{C} 4-\mathrm{C} 3$, respectively. The bond angles of $\mathrm{C} 1-\mathrm{C} 2-\mathrm{C} 3$ and $\mathrm{C} 2-\mathrm{C} 3-\mathrm{C} 4$ are $124.0(1)^{0}$ and $122.5(1)^{0}$, respectively. The torsion angle of the carbon skeleton (C4-C3-C2-C1) is $59.8(2)^{0}$. Cyclohexyl ring (plane $2=\mathrm{C} 5-\mathrm{C} 6-\mathrm{C} 7-\mathrm{C} 8-\mathrm{C} 9-\mathrm{C} 10$ ) is in chair conformation and mean deviation from plane is $0.2374 \AA$. The piperazine ring (plane $3=\mathrm{N} 2-\mathrm{C} 11-\mathrm{C} 12-\mathrm{N} 3-$ C13-C14) is also in chair conformation and mean deviation from plane is $0.0004 \AA$. Dihedral angles are 70.695 between planes 2 and 3 .

Table 1. Crystallographic data and structure refinement for compound $\mathbf{4 g}$

\begin{tabular}{|c|c|}
\hline Sum formula & $\mathrm{C}_{17} \mathrm{H}_{24} \mathrm{~N}_{3} \mathrm{O}_{4} \mathrm{Cl}_{3} \mathrm{~S}$ \\
\hline $\mathrm{f}_{\mathrm{w}}, \mathrm{g} \cdot \mathrm{mol}^{-1}$ & 472.81 \\
\hline Crystal dimensions, $\mathrm{mm}$ & $0.50 \times 0.40 \times 0.20 \mathrm{~mm}$ \\
\hline Crystal system & orthorhombic \\
\hline Space group & Pbca (\#61) \\
\hline Lattice Parameters & \\
\hline$a(\AA)$ & $9.28220(10)$ \\
\hline$a(\AA)$ & $21.3903(3)$ \\
\hline$c \quad(\AA)$ & $21.9341(3)$ \\
\hline $\operatorname{Vol}\left[\AA^{3}\right]$ & $4354.99(10)$ \\
\hline $\mathrm{Z}$ & 8 \\
\hline$D_{\text {calc. }} . \mathrm{g} \cdot \mathrm{cm}^{-3}$ & 1.442 \\
\hline$\mu, \mathrm{cm}^{-1}$ & 5.44 \\
\hline$F(000)$ & 1968.00 \\
\hline Index ranges & $-13<=h<=13$ \\
\hline & $-30<=k<=28$ \\
\hline & $-30<=l<=30$ \\
\hline Reflections colle & 247006 \\
\hline Independent reflections & $7079\left(\mathrm{R}_{\mathrm{int}}=0.023\right)$ \\
\hline Data / restraints / parameters & $6174 / 0 / 277$ \\
\hline Goodness-of-fit on $F^{2}$ & 1.118 \\
\hline Final $R$ indices $[I>3 \sigma(\mathrm{I})]$ & $\mathrm{R}=0.072, \mathrm{wR}=0.033$ \\
\hline Largest diff. peak and hole & 0.43 and -0.54 e. $\AA^{-3} /$ \\
\hline \multicolumn{2}{|c|}{$R=\Sigma I I F o l-l F c l l / \Sigma l F o l, R_{w}=\left[\Sigma w(l F o l-l F c l)^{2} / \Sigma w F_{0}^{2}\right]^{1 / 2}$} \\
\hline
\end{tabular}

Figure 2. ORTEP view of the molecular structure of $\mathbf{4 g}$ in the crystal; displacement ellipsoids are drawn at the $50 \%$ probability level 
Table 2. Selected bond lengths $[\AA]$ and angles $\left[{ }^{\circ}\right]$ for compound $\mathbf{4 g}$

\begin{tabular}{llllllll}
\hline \multicolumn{5}{c}{ Bond Lengths } & \multicolumn{5}{c}{ Angles } \\
\hline $\mathrm{S}(1) \mathrm{C}(4)$ & $1.755(1)$ & $\mathrm{S}(1) \mathrm{C}(5)$ & $1.841(1)$ & $\mathrm{C}(4) \mathrm{S}(1) \mathrm{C}(5)$ & $106.37(7)$ & $\mathrm{O}(2) \mathrm{N}(1) \mathrm{O}(1)$ & $122.0(1)$ \\
$\mathrm{Cl}(1) \mathrm{C}(1)$ & $1.724(2)$ & $\mathrm{Cl}(3) \mathrm{C}(2)$ & $1.746(1)$ & $\mathrm{O}(2) \mathrm{N}(1) \mathrm{C}(3)$ & $119.9(1)$ & $\mathrm{O}(1) \mathrm{N}(1) \mathrm{C}(3)$ & $118.0(1)$ \\
$\mathrm{Cl}(2) \mathrm{C}(1)$ & $1.705(1)$ & $\mathrm{N}(1) \mathrm{O}(2)$ & $1.241(2)$ & $\mathrm{C}(4) \mathrm{N}(2) \mathrm{C}(11)$ & $124.2(1)$ & $\mathrm{C}(4) \mathrm{N}(2) \mathrm{C}(14)$ & $122.4(1)$ \\
$\mathrm{N}(1) \mathrm{O}(1)$ & $1.235(2)$ & $\mathrm{N}(1) \mathrm{C}(3)$ & $1.415(2)$ & $\mathrm{C}(11) \mathrm{N}(2) \mathrm{C}(14) 112.9(1)$ & $\mathrm{C}(12) \mathrm{N}(3) \mathrm{C}(13) 113.5(1)$ \\
$\mathrm{N}(2) \mathrm{C}(4)$ & $1.335(2)$ & $\mathrm{N}(2) \mathrm{C}(11)$ & $1.468(2)$ & $\mathrm{C}(12) \mathrm{N}(3) \mathrm{C}(15)$ & $120.7(1)$ & $\mathrm{C}(13) \mathrm{N}(3) \mathrm{C}(15) 125.0(1)$ \\
$\mathrm{N}(2) \mathrm{C}(14)$ & $1.468(2)$ & $\mathrm{N}(3) \mathrm{C}(12)$ & $1.451(2)$ & $\mathrm{C}(1) \mathrm{C}(2) \mathrm{C}(3)$ & $124.0(1)$ & $\mathrm{C}(1) \mathrm{C}(2) \mathrm{Cl}(3)$ & $118.3(1)$ \\
$\mathrm{N}(3) \mathrm{C}(13)$ & $1.453(2)$ & $\mathrm{N}(3) \mathrm{C}(15)$ & $1.345(2)$ & $\mathrm{C}(3) \mathrm{C}(2) \mathrm{Cl}(3)$ & $117.6(1)$ & $\mathrm{C}(3) \mathrm{C}(4) \mathrm{S}(1)$ & $114.8(1)$ \\
$\mathrm{C}(2) \mathrm{C}(1)$ & $1.326(2)$ & $\mathrm{C}(2) \mathrm{C}(3)$ & $1.454(2)$ & $\mathrm{C}(3) \mathrm{C}(4) \mathrm{N}(2)$ & $123.5(1)$ & $\mathrm{S}(1) \mathrm{C}(4) \mathrm{N}(2)$ & $121.6(1)$ \\
$\mathrm{O}(3) \mathrm{C}(15) 1.212(2)$ & $\mathrm{C}(4) \mathrm{C}(3)$ & $1.400(2)$ & $\mathrm{C}(11) \mathrm{C}(12) \mathrm{N}(3) 109.1(1)$ & $\mathrm{Cl}(1) \mathrm{C}(1) \mathrm{Cl}(2) 113.61(8)$ \\
$\mathrm{C}(12) \mathrm{C}(11) 1.516(2)$ & $\mathrm{O}(4) \mathrm{C}(15)$ & $1.336(2)$ & $\mathrm{Cl}(1) \mathrm{C}(1) \mathrm{C}(2)$ & $121.0(1)$ & $\mathrm{Cl}(2) \mathrm{C}(1) \mathrm{C}(2)$ & $125.4(1)$ \\
$\mathrm{O}(4) \mathrm{C}(16) 1.443(4)$ & $\mathrm{C}(6) \mathrm{C}(5)$ & $1.528(2)$ & $\mathrm{N}(2) \mathrm{C}(11) \mathrm{C}(12) 111.1(1)$ & $\mathrm{C}(15) \mathrm{O}(4) \mathrm{C}(16) 115.9(2)$ \\
$\mathrm{C}(6) \mathrm{C}(7)$ & $1.520(2)$ & $\mathrm{C}(5) \mathrm{C}(10)$ & $1.529(2)$ & $\mathrm{C}(5) \mathrm{C}(6) \mathrm{C}(7)$ & $110.4(1)$ & $\mathrm{N}(1) \mathrm{C}(3) \mathrm{C}(2)$ & $116.2(1)$ \\
$\mathrm{C}(7) \mathrm{C}(8)$ & $1.523(2)$ & $\mathrm{C}(13) \mathrm{C}(14)$ & $1.517(2)$ & $\mathrm{N}(1) \mathrm{C}(3) \mathrm{C}(4)$ & $121.4(1)$ & $\mathrm{C}(2) \mathrm{C}(3) \mathrm{C}(4)$ & $122.5(1)$ \\
$\mathrm{C}(8) \mathrm{C}(9)$ & $1.507(3)$ & $\mathrm{C}(10) \mathrm{C}(9)$ & $1.524(3)$ & $\mathrm{C}(10) \mathrm{C}(5) \mathrm{S}(1)$ & $107.6(1)$ & $\mathrm{C}(10) \mathrm{C}(5) \mathrm{C}(6)$ & $110.3(1)$ \\
$\mathrm{C}(16)(17)$ & $1.263(7)$ & & & $\mathrm{S}(1) \mathrm{C}(5) \mathrm{C}(6)$ & $111.4(1)$ & $\mathrm{C}(8) \mathrm{C}(7) \mathrm{C}(6)$ & $110.7(1)$ \\
& & & & $\mathrm{C}(14) \mathrm{C}(13) \mathrm{N}(3) 110.7(1)$ & $\mathrm{N}(2) \mathrm{C}(14) \mathrm{C}(13) 109.5(1)$ \\
& & & & $\mathrm{C}(9) \mathrm{C}(8) \mathrm{C}(7)$ & $110.3(2)$ & $\mathrm{N}(3) \mathrm{C}(15) \mathrm{O}(3)$ & $124.6(2)$ \\
& & & & $\mathrm{C}(3) \mathrm{C}(15) \mathrm{O}(4)$ & $110.8(2)$ & $\mathrm{O}(3) \mathrm{C}(15) \mathrm{O}(4)$ & $124.5(2)$ \\
& & & & $\mathrm{C}(17) \mathrm{C}(16) \mathrm{C}(4)$ & $110.6(1)$ & $\mathrm{C}(8) \mathrm{C}(9) \mathrm{C}(10)$ & $111.9(2)$ \\
\hline
\end{tabular}

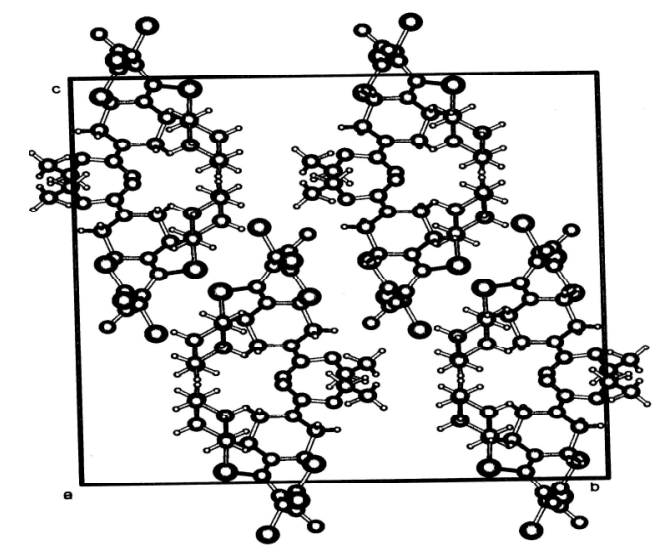

Figure 3. Packing diagram of $\mathbf{4 g}$; molecular overlap view from the a-axis

Suitable single crystal of $\mathbf{4 g}$ was obtained by slow evaporation of an ethanol solution. A single crystal of $\mathbf{4 g}$ was mounted on an Rigaku R-Axis Rapid-S diffractometer equipped with a graphite monochromatized MoK $\alpha$ radiation source $(\lambda=0.71073 \AA)$. The data were collected at a temperature of $20 \pm 1^{\circ} \mathrm{C}$ to a maximum $2 \theta$ value of $60.2^{0}$. The structures were solved $^{42}$ by SIR 92 and refined with CRYSTALS ${ }^{43}$. The positions of the $\mathrm{H}$ atoms bonded to $\mathrm{C}$ atoms were calculated $(\mathrm{C}-\mathrm{H}$ distance $0.95 \AA$ ) and refined using a riding model. The $\mathrm{H}$ atom displacement parameters were restricted to be $1.2 \mathrm{Ueq}$ of the parent atom. All calculations were carried out using the crystal structure analysis software package ${ }^{44}$. ORTEP-III view of the molecular structure ${ }^{45}$ of $\mathbf{4 g}$ is given in Figure 2. Crystallographic data (excluding structure factors) for the structure reported in this paper have been deposited with the Cambridge Crystallographic Data Centre as supplementary publication no. CCDC717717 for $\mathbf{4 g}^{46}$. 


\section{Conclusion}

Our investigations started with the synthesis of pentachloro-2-nitro-1,3-butadiene $(\mathbf{1})^{28}$ which was obtained in three steps from trichloroethylene and it serves as starting material for new $N, S$-substituted nitrodiene compounds. Nitrodienes and especially their halogen derivatives is primarily due to the possibilities of their application as important intermediate products for the synthesis of various heterocyclic compounds. From the spectroscopic data and related literature it can be concluded that these new compounds are formed with vinylic nucleophilic substitution. As can be seen from literature data, these compounds also serve as useful precursors for the synthesis of physiologically active substances. The aim in this study was to synthesize and characterize new $N, S$-substituted nitrodiene compounds and to determine the crystal structures of representative products. The products of these novel compounds were characterized by elemental analysis, FT-IR, ${ }^{1} \mathrm{H}-\mathrm{NMR},{ }^{13} \mathrm{C}-\mathrm{NMR}$ and mass spectroscopies. The new $N, S$-substituted nitrobutadienes are obtained in good yields. These are stable and yellow coloured compounds. Further biological testing is underway.

\section{Acknowledgment}

We gratefully acknowledge the Research Fund of the University of Istanbul for financial support for this work.

\section{References}

1. Zapol'skii V A, Namyslo J C, Altug C, Gjikaj M and Kaufmann D E, Synthesis, 2008, 304.

2. Zapol'skii V A, Namyslo J C, Gjikaj M and Kaufmann D E, ARKIVOC, 2007, 1, 76-93.

3. Zapol'skii V A, Namyslo J C, Gjikaj M and Kaufmann D E, Synlett., 2007, 1507-1512.

4. Kaberdin R V, Potkin V I and Zapols'kii V A, Russ Chem Rev., 1997, 66, 827.

5. Potkin V I, Zapol'skii V A, Knizhnikov V A, Kaberdin R V, Yanuchok A A and Petkevich S K, Russ J Org Chem., 2001, 37, 689-694.

6. Potkin V I, Zapol'skii V A, Knizhnikov V A and Kaberdin R V, Russ J Org Chem., 2000, 36, 877-883.

7. Nechai N I, Potkin V I and Kaberdin R V, Russ J Org Chem., 2000, 36, 650.

8. Henry Bluestone, Diamond Alkali CO, US3021370 (13.02.1962)

9. Fischer Reiner (De) and Jeschke Peter (De) Bayer Cropscience AG (De), WO03040129, (15.05.2003)

10. Nishiyoma M, Yamamoto T and Koie Y, Tetrahedron Lett., 1998, 39, 617.

11. Kerrigon F, Martin C and Thomas G H, Tetrahedron Lett., 1998, 39, 2219.

12. Solodin I and Heath T D, Synlett., 1996, 7, 619.

13. Baltzly R, Ide W S and Lorz E, J Am Chem Soc., 1955, 77, 4809.

14. Harfenist M, J Am Chem Soc., 1957, 79, 2211.

15. Smith D R, Curry J W and Eifert R L, J Am Chem Soc., 1950, 72, 2969.

16. İbiş C and Aydınlı G, Phosphorus Sulfur Silicon, 2004, 179, 1975-1982.

17. İbiș C and Gökmen Z, Phosphorus Sulfur Silicon, 2004, 179, 2537.

18. İbiş C, Kırbaşlar F G and Aydınlı G, Phosphorus Sulfur Silicon, 2005, 180, 365.

19. İbiş C and Gökmen Z, Phosphorus Sulfur Silicon, 2006, 181, 939.

20. İbiş C, Sayıl Ç and Özkök F, Z Naturforsch., 2006, 61b, 1174.

21. İbiş C, Kırbaşlar F G and Aydınlı G, Phosphorus Sulfur Silicon, 2006, 181, 573.

22. İbiş C and Deniz N G, Indian J Chem., 2007, 46B, 674.

23. İbiş C and Gökmen Z, Rev Roum Chim., 2007, 52, 957. 
24. İbiş C and Onul N Y, Rev Roum Chim., 2007, 52, 953.

25. İbiş C and Aydınlı G, Phosphorus Sulfur Silicon, 2007, 182, 1427-1436.

26. İ̉iş C and Sayıl Ç Phosphorus Sulfur Silicon, 1995, 106, 29.

27. Ol'dekop Yu A, Kaberdin R V and Potkin V I, Zh Org Khim., 1980, 16, 543.

28. Ol'dekop Yu A and Kaberdin R V, Zh Org Khim., 1976, 12, 2039.

29. Kaberdin R V and Potkin V I, Polikhlor-1,3-butadieny, Polychloro-1,3-butadienes, Nauka i Tekhnica, 1991, Minsk.

30. Kokorev V N, Potkin V I, Kaberdin R V and Ol'dekop Yu A, Izv Akad Nauk Bel SSR, Ser Khim Nauk., 1982, 2, 17.

31. Kokorev V N, Potkin V I, Kaberdin R V and Ol'dekop Yu A, Izv Akad Nauk Bel SSR, Ser Khim Nauk., 1987, 3, 62.

32. Potkin V I, Zelenkovskii V M, Zapol'skii V A, Shingel I A and Kaberdin R V, Izv Akad Nauk Bel SSR Ser Khim Nauk, 1995, $2,71$.

33. Zelenkovskii V M, Potkin V I and Kaberdin R V, Dokl Akad Nauk Belarusi., 1999, 43, 56.

34. Surange S S, Kumaran G, Rajappa S, Rajalaksmi K and Pattabhi V, Tetrahedron, 1997, 53, 8531.

35. İbiş C, Sayıl M C and Deniz N G, Acta Cryst., 2006, E62, o800.

36. İbiş C, Sayıl M C and Ozkok F, Acta Cryst., 2006, E62, o1147.

37. İbiş C and Deniz N G, Acta Cryst., 2006, E62, o5373.

38. İbiş C and Gokmen Z, Acta Cryst., 2006, E62, o2932.

39. İbiş C and Deniz N G, Acta Cryst., 2007, E63, o3058.

40. İbiş C and Deniz N G, Acta Cryst., 2007, E63, o1091.

41. Aydınlı G, Sayıl C and İbiş C, Acta Cryst., 2009, E65, o272.

42. Altomare A, Cascarano G, Giacovazzo C, Guagliardi A, Burla M C, Polidori G and Camalli M, J Appl Cryst., 1994, 27, 435-436.

43. Watkin D J, Prout C K, Carruthers J R and Betteridge P W, CRYSTALS, Issue 10, Chemical Crystallography Laboratory, Oxford, 1996, UK.

44. Crystal Structure 3.5.1, Crystal Structure Analysis Package, Rigaku and Rigaku/MSC 9009 New Trails Dr. The Woodlands, USA, 2000-2003, TX 77381.

45. Farrugia L J, J Appl Cryst., 1997, 30, 565.

46. Cambridge Crystallographic Data Center (CCDC), 12 Union Road, Cambridge CB2 1EZ, UK, by quoting the depository number CCDC-717717. 


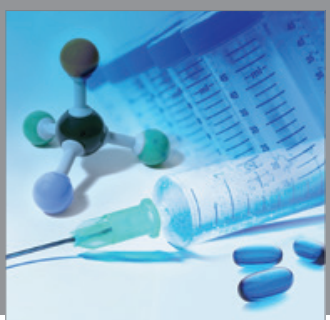

International Journal of

Medicinal Chemistry



Carbohydrate Chemistry

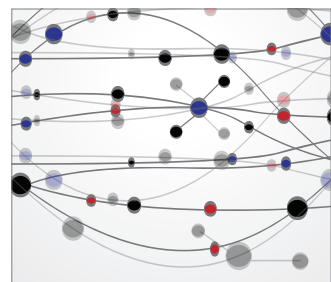

The Scientific World Journal
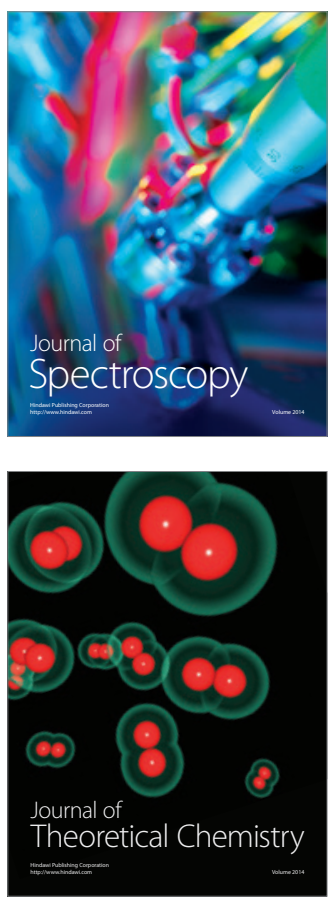
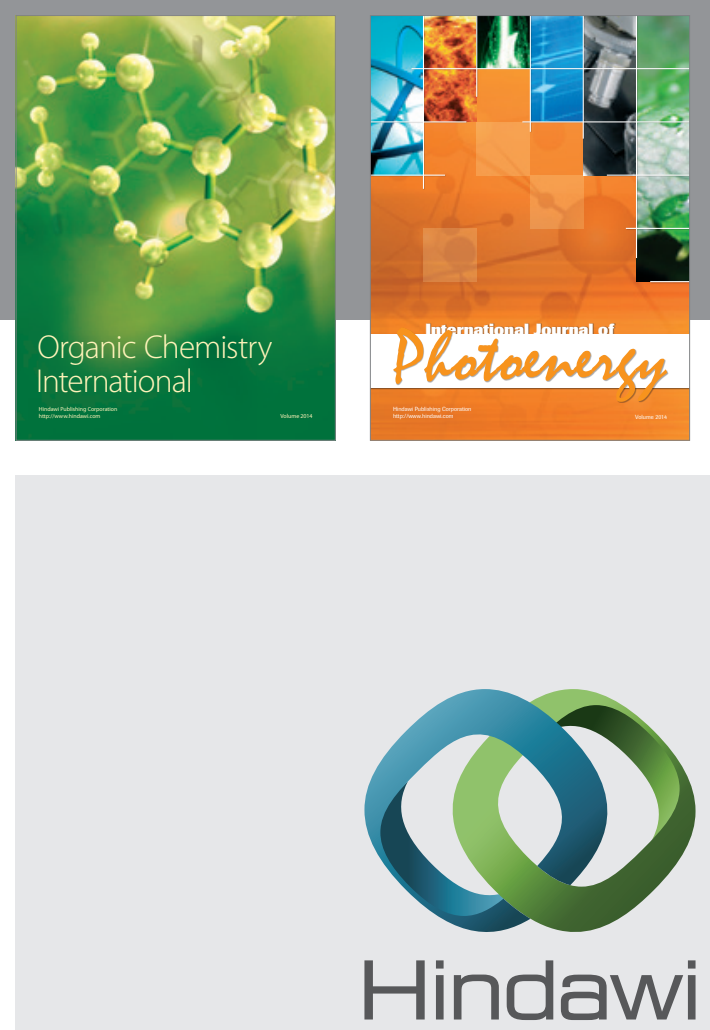

Submit your manuscripts at

http://www.hindawi.com
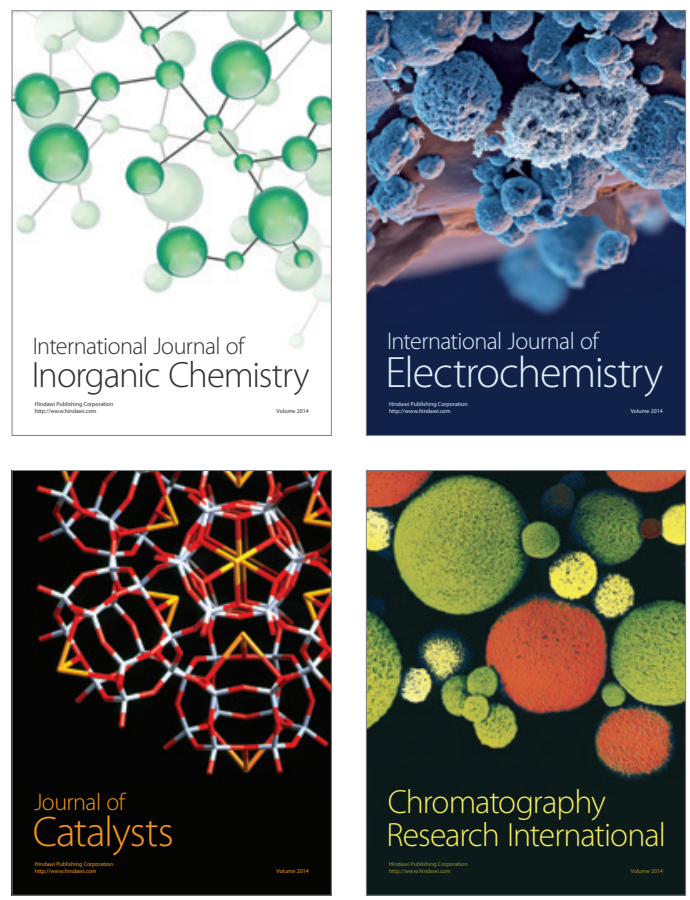
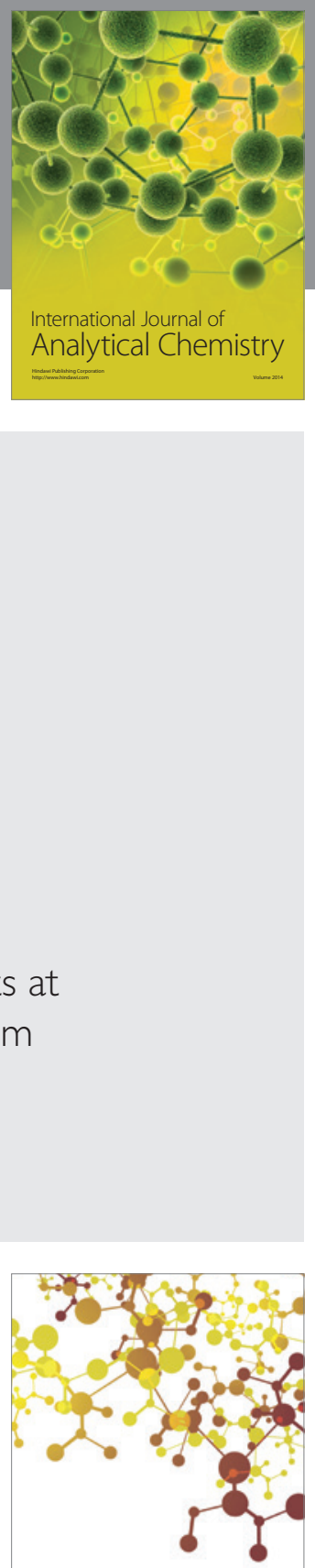

Journal of

Applied Chemistry
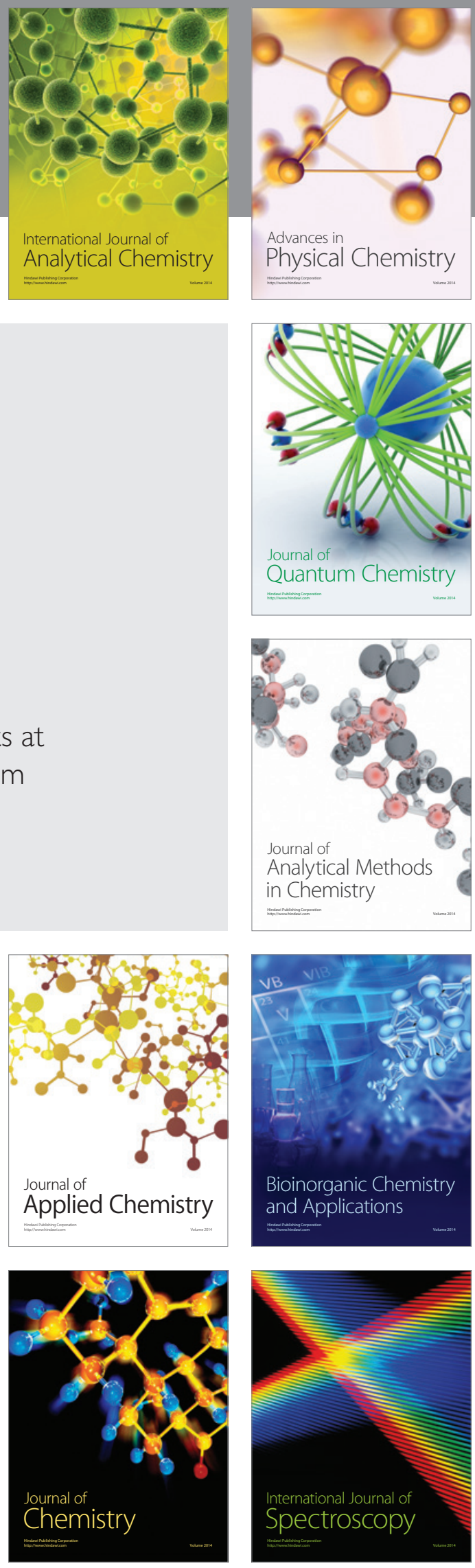